\title{
Improving the Understanding of Provenance and Reproducibility of a Multi-Sensor Merged Climate Data Record
}

\author{
Hook Hua, Brian Wilson, Gerald Manipon, Lei Pan, and Eric Fetzer \\ Jet Propulsion Laboratory, California Institute of Technology \\ 4800 Oak Grove Drive, Pasadena, California 91109, U.S.A \\ \{hook.hua, bdwilson, gmanipon, lei.pan, eric.j.fetzer\}@jpl.nasa.gov
}

\begin{abstract}
Multi-decadal climate data records are critical to studying climate variability and change. These often also require merging data from multiple instruments such as those from NASA's A-Train that contain measurements covering a wide range of atmospheric conditions and phenomena. Multidecadal climate data record of water vapor measurements from sensors on ATrain, operational weather, and other satellites are being assembled from existing data sources, or produced from well-established methods published in peer-reviewed literature. However, the immense volume and inhomogeneity of data often requires an "exploratory computing" approach to product generation where data is processed in a variety of different ways with varying algorithms, parameters, and code changes until an acceptable product is generated. Furthermore, the data product information associated with source data, processing methods, parameters used, intermediate $\&$ final product outputs, and associated materials are often hidden in each of the trials and scattered throughout the processing system(s). We will present methods to help users better capture and explore the production legacy of the data, metadata, ancillary files, code, and computing environment changes used during the production of these merged and multi-sensor data products. By building provenance services on semantic and provenance technologies, we show how to leverage provenance-as-a-service to capture sufficient information to enable users to track processing, perform faceted searches on the provenance record, and visualize the provenance of the products and processing lineage. We will also present services for capturing sufficient provenance information and the associated artifacts to enable some reproducibility of these climate data records.
\end{abstract}

Keywords: provenance, semantic, open provenance model, reproducibility, multi-sensor, data fusion, climate data, web services, faceted navigation, visualization. 